\title{
AKTUELLE EINFÜHRUNGEN IN DAS FACH DEUTSCH ALS FREMDSPRACHE: didaktisch-methodische Wissensordnungen im Umbruch
}

\author{
Dörthe Uphoff \\ (Universidade de São Paulo) \\ https://orcid.org/0000-0002-1795-8659
}

\section{ZUSAMMENFASSUNG}

In diesem Artikel möchte ich untersuchen, wie die Methodengeschichte und die aktuelle didaktisch-methodische Diskussion in fünf Einführungswerken für Deutsch als Fremdsprache $(\mathrm{DaF})$ dargestellt werden, die zwischen 2012 und 2018 veröffentlicht wurden (RÖSLER, 2012; HUNEKE \& STEINIG, 2013; ROCHE, 2013; BRINITZER et al., 2016; GEHRING, 2018). Ich gehe dabei von der Annahme aus, dass in der Geschichte des Fremdsprachenunterrichts im 20. Jahrhundert die Suche nach der besten Methode im Vordergrund stand, während sich zu Beginn des 21. Jahrhunderts ein Paradigmenwechsel hin zu einer Post-Methoden-Pädagogik abzeichnet (vgl. KUMARAVADIVELU, 2006). Um zu erforschen, wie dieser Moment des Übergangs von ExpertInnen aus dem DaF-Bereich erzählt wird, mobilisiere ich die in Brasilien noch wenig bekannte Wissenssoziologische Diskursanalyse (KELLER 2011a, 2011b), welche die Konstruktion, Legitimation und Transformation von Wissensordnungen aus einem Blickwinkel beleuchtet, der die Foucaultsche Diskursperspektive mit der Wissenssoziologie von Berger \& Luckmann (1966) verbindet. Die Analyse belegt die Existenz unterschiedlicher diskursiver Strategien in den Einführungswerken, sowohl im Hinblick auf die Darstellung der Methodengeschichte als auch auf die Einschätzung der aktuellen didaktisch-methodischen Situation, was auf Wissensordnungen im Umbruch in der derzeitigen akademischen Diskussion schließen lässt.

SCHLÜSSELWÖRTER: Deutsch als Fremdsprache; Wissenssoziologische Diskursanalyse; Post-Methoden-Ära 


\section{OBRAS INTRODUTÓRIAS ATUAIS NA ÁREA DE ALEMÃO COMO LÍNGUA ESTRANGEIRA: regimes de saber didático-metodológico em transformação}

\section{RESUMO}

O artigo tem por objetivo examinar como a história e atual discussão didático-metodológica são apresentadas em cinco obras de introdução à área de Alemão como Língua Estrangeira (Deutsch als Fremdsprache; DaF) publicadas entre 2012 e 2018 (RÖSLER, 2012; HUNEKE \& STEINIG, 2013; ROCHE, 2013; BRINITZER et al., 2016; GEHRING, 2018). Parte-se do pressuposto de que a história do ensino de línguas no século XX costuma ser vista pelo ângulo da busca do melhor método, enquanto que no início do século XXI observa-se uma mudança de paradigma em direção a uma pedagogia pós-método (cf. KUMARAVADIVELU, 2006). No intuito de investigar como esse momento de transição é narrado por estudiosos da área de DaF, mobilizamos como referencial teórico a abordagem discursiva SKAD (Sociology of Knowledge Approach to Discourse; KELLER 2011a, 2011b), ainda pouco conhecida no Brasil, que investiga os processos de construção, legitimação e transformação de campos de saberes sob uma ótica que aproxima a perspectiva foucaultiana à sociologia do conhecimento de Berger \& Luckmann (1966). A análise do corpus revela a presença de estratégias discursivas divergentes nas obras, tanto no que diz respeito à apresentação do passado quanto à avaliação do cenário didático-metodológico recente, apontando para regimes de saber em transformação na discussão acadêmica atual.

PALAVRAS-CHAVE: Alemão como Língua Estrangeira; análise do discurso com foco na sociologia do conhecimento; era pós-método 


\section{CURRENT INTRODUCTORY BOOKS TO GERMAN AS A FOREIGN LANGUAGE: didactic-methodological knowledge regimes in transition}

\section{ABSTRACT}

The article aims to investigate how the history and the current didactic-methodological discussion are presented in five introductory books to German as a Foreign Language (Deutsch als Fremdsprache, DaF) published between 2012 and 2018 (RÖSLER, 2012; HUNEKE \& STEINIG, 2013; ROCHE, 2013; BRINITZER et al., 2016; GEHRING, 2018). We start from the assumption that the $20^{\text {th }}$ century history of language teaching usually focusses on the search for the best method, while in the beginning of the $21^{\text {st }}$ century there is a paradigm shift towards a post-method pedagogy (cf. KUMARAVADIVELU, 2006). In order to investigate the narration of this transitional moment by scholars in the field of DaF, we adopt concepts of the Sociology of Knowledge Approach to Discourse (SKAD) by Keller (2011a, 2011b), which is still relatively unknown in Brazil. SKAD investigates the processes of construction, legitimation and transformation of knowledge fields from an angle that combines the Foucaultian perspective on discourse with the sociology of knowledge presented by Berger and Luckmann (1966). The analysis of the corpus reveals the presence of divergent discursive strategies in the introductory books, both with regard to the presentation of the past and the evaluation of the recent didactic-methodological scenario, pointing to changing knowledge regimes in the current academic discussion.

Keywords: German as a Foreign Language; Sociology of Knowledge Approach to Discourse; Postmethod Era 


\section{Einleitung}

In diesem Beitrag möchte ich untersuchen, wie der aktuelle didaktisch-methodische Wissensstand im Bereich Deutsch als Fremdsprache $(\mathrm{DaF})$ in einschlägigen Einführungswerken dargestellt wird. Meine Arbeitshypothese ist dabei, dass sich - gemäß des Untertitels „From method to postmethod" des einflussreichen Werkes von Kumaravadivelu (2006) - die didaktisch-methodische Diskussion im Fremdsprachenunterricht in den letzten zwanzig Jahren grundlegend verschoben hat. Während im 20. Jahrhundert die Suche nach einer allgemeingültigen, „besten“ Methode im Vordergrund stand, wird heute davon ausgegangen, dass es einen solchen universell überlegenen Vermittlungsweg nicht gibt und stattdessen nur lokale Lösungen für die Herausforderungen des Lehrens und Lernens von Fremdsprachen gefunden werden können. Orientierungen für diesen „kontextsensiblen Fremdsprachenunterricht“ - so der Titel eines vor kurzem erschienenen Buches von Gerlach und Leupold (2019) - geben allgemeine lernpädagogische Prinzipien, deren konkrete Ausgestaltung jedoch von der jeweiligen Unterrichtskultur und den Kontextfaktoren abhängen. Dieser Perspektivenwechsel muss nun aber, wie Leffa (2012) betont, auch mit einem Wandel der Kräfteverhältnisse im Fremdsprachenunterricht einhergehen:

O ensino de línguas não deve acontecer em um mundo abstrato, meramente teórico e construído por autoridade, seja no sentido autoritário, com base no poder, seja no sentido autorizado, com base no saber, mas deve estar situado em um determinado contexto, com base na realidade, garantindo ao professor a opção de agir dentro daquilo que é plausível em seu contexto [...] (LEFFA, 2012, p. 399).

In dieser Einschätzung klingen neben der particularity auch die Parameter der practicality und possibility von Kumaravadivelu (2003, 2006) an, welche einfordern, dass ein kontextsensibler Fremdsprachenunterricht der Lehrkraft mehr eigenständiges pädagogisches Urteilsvermögen und damit methodisch-didaktische Entscheidungsfreiheit zugestehen muss, als dies im 20. Jahrhundert im Rahmen der globalen Methodenkonzeptionen vorgesehen war. Wie wird diese Bewegung hin zu einem grundlegenden Paradigmenwechsel in der Fremdsprachendidaktik nun im Bereich Deutsch als Fremdsprache wahrgenommen? Werden die Schriften von Kumaravadivelu überhaupt 
rezipiert oder werden stattdessen vielleicht andere Entwicklungen in der wissenschaftlichen Diskussion priorisiert? Um diese Fragen zu beantworten, schlage ich einen wissenssoziologischen Blick auf die Disziplin DaF vor, in dessen Rahmen die Produktion von Wissen eine besondere Form des sozialen Handelns und der Kommunikation darstellt (vgl. WEINGART, 2003, p. 12). Unter dieser Perspektive verstehe ich ein wissenschaftliches Fachgebiet (z.B. DaF) als eine spezialisierte Kommunikationsgemeinschaft, die ihre neuen Mitglieder durch spezifische Sozialisationsprozesse (Kurse, Studiengänge) und instrumente (Fachliteratur) ausbildet und formt (WEINGART, 2003, p. 44). Einführende Lehrbücher können nach dieser Sichtweise als eine Zusammenstellung des zu einem bestimmten Zeitpunkt legitimierten und von der scientific community mehrheitlich anerkannten Wissenskanons gewertet werden. Deshalb sollen sie in dieser Arbeit herangezogen werden, um zu analysieren, wie die aktuelle didaktisch-methodische Diskussion in Deutsch als Fremdsprache dargestellt wird und ob insbesondere auch von einer Zäsur im beginnenden 21. Jahrhundert gesprochen wird. $\mathrm{Zu}$ diesem Zweck möchte ich im Folgenden fünf Einführungen für DaF, die im Zeitraum von 2012 bis 2018 in Deutschland publiziert worden sind, genauer untersuchen: Rösler (2012), Huneke \& Steinig (2013), Roche (2013), Brinitzer et al. (2016), Gehring (2018). Es handelt sich dabei um die aktuellsten Werke in diesem Bereich, die sich darüber hinaus auch durch ihr handliches Format auszeichnen, d.h. eine maximale Länge von ca. 300 Seiten nicht überschreiten, welche ihren kompakten und zusammenfassenden Charakter unterstreicht. Die Bezeichnung „Deutsch als Fremdsprache“ für das betreffende Fachgebiet - im Vergleich zu „Deutsch als Zweitsprache“ oder „língua adicional", im brasilianischen Sprachgebrauch, - ist dabei nicht trivial und muss in der nächsten Sektion zunächst genauer erläutert werden. Die nachfolgenden Sektionen 3 und 4 geben einen Einblick in den theoretischen Rahmen der Wissenssoziologischen Diskursanalyse nach Keller (2011a, 2011b), sowie in die spezifische Analysekategorie der Narration in diesem Kontext (VIEHÖVER, 2006, 2010, 2012), welche für unsere Untersuchung herangezogen werden soll. Beide Konzepte sind in Brasilien - wie auch im DaF-Bereich an sich - bisher kaum mobilisiert worden, stellen jedoch aus meiner Sicht ein übergreifendes, interdisziplinäres Forschungsprogramm dar, das bisher wenig beachtete Aspekte in der DaF-didaktischen Diskussion zum Vorschein 
bringen kann. Sektion 5 präsentiert die unterschiedlichen diskursiven Narrationsstrategien, anhand derer der aktuelle methodische Wissensstand für $\mathrm{DaF}$ in den Einführungswerken dargestellt bzw. „erzählt“ wird. Ein paar abschließende Bemerkungen zur DaF-Lehrerbildung in diesem Zusammenhang sollen den Artikel beenden.

\section{Deutsch als Fremdsprache}

In der brasilianischen Diskussion zum Fremdsprachenunterricht wird in den letzten Jahren immer häufiger für den Begriff „língua

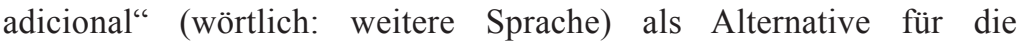
Bezeichnungen „língua estrangeira“ und „segunda língua“ plädiert, da diese, so die Kritik, einen fundamentalen Unterschied in den beiden benannten Aneignungssituationen suggerieren, der aber oft nicht eindeutig und trennscharf zu identifizieren sei (cf. SCHLATTER; GARCEZ, 2009; LEFFA; IRALA 2014).

Auch in der DaF-Literatur gibt es Bedenken bezüglich der Begriffe der Fremd- und Zweitsprache. So warnt z.B. Rösler (2012, p. 30) davor, die Opposition zwischen Zweit- und Fremdsprache unkritisch mit den Begriffspaaren „natürlich vs. gesteuert“ bzw. „Lernort innerhalb oder außerhalb des deutschsprachigen Raums" gleichzusetzen. Als Definition, so der Autor (idem, p. 31), könne jedoch gelten,

dass Deutsch als Zweitsprache überwiegend im deutschsprachigen Raum stattfindet und gesteuert und natürlich erworben wird, während Deutsch als Fremdsprache überwiegend gesteuert erworben wird und innerhalb und außerhalb des deutschsprachigen Raums stattfinden kann.

Darüber hinaus sei auch ein weiteres Bestimmungselement besonders wichtig: „Wer Deutsch als Zweitsprache lernt, verwendet diese Sprache gleichzeitig in seiner Lebenswelt. Sie ist für seinen Alltag unmittelbar relevant" (idem; Hervorhebung im Original). Damit betont Rösler, dass es einen konzeptuellen Unterschied zwischen Zweit- und Fremdsprache gibt, der jedoch nur indirekt - im Sinne einer höheren Rate der Wahrscheinlichkeit - mit den Kriterien des geographischen Raumes und der institutionellen Steuerung des Lernprozesses korreliert und eher von der subjektiven Bedeutung der Sprache für die Lebenswelt der Lernenden abhängt.

Man könnte nun einwerfen, dass auch eine ,unmittelbare Relevanz für den Alltag“ noch nicht eindeutig genug den zweitsprachlichen 
Erwerbskontext beschreibt. So argumentieren Schlatter und Garcez (2009, p. 127-128), dass auch transnationale Sprachen wie Spanisch und Englisch, die in der globalen Kommunikation stark präsent sind, in der Lebenswelt brasilianischer Lernender ihre Spuren hinterlassen und deshalb mehr als „nur“ Fremdsprachen darstellen:

De fato, se consideramos que o espanhol e o inglês constituem patrimônios relevantes para a formação do cidadão a ponto de nos ocuparmos do seu cultivo na educação nacional, entendemos que, de alguma maneira, essas línguas fazem parte dos recursos necessários para a cidadania contemporânea. Nesse sentido, são línguas adicionais, úteis e necessárias entre nós, não necessariamente estrangeiras. Assim, falar de uma língua adicional em vez de língua estrangeira enfatiza o convite para que os educandos (e os educadores) usem essas formas de expressão para participar na sua própria sociedade (SCHLATTER; GARCEZ, 2009, p. 128).

Die Bedeutung von Kenntnissen anderer Sprachen wird hier also mit einer bürgerpolitischen Erziehung in Verbindung gebracht und so die Relevanz ,weiterer“ Sprachen für die Lebenswelt auch derjeniger Schülerinnen und Schüler begründet, die in ihrem sonstigen Alltag nur wenig Gelegenheit haben, eine Fremdsprache aktiv zu benutzen. Der Fremdsprachenunterricht erhält damit eine innenpolitische Tragweite und wird als ein Mittel gesehen, soziale Ungerechtigkeiten in Brasilien abzubauen. So formulieren Leffa und Irala (2014) eindrücklich:

Quando se fala em língua adicional, defende-se também a ideia de que seu ensino é um direito individual do aluno com benefícios para a coletividade. O domínio de outra(s) língua(s) deixou de ser um luxo, concedido a poucos privilegiados com oportunidade de viajar para o exterior, para se tornar um direito de todos e uma prioridade nacional. Negar ao aluno o acesso a uma língua adicional não é apenas uma maneira de excluí-lo, com prejuízo para o exercício de sua cidadania; é também uma maneira de retardar o desenvolvimento de um país no mundo contemporâneo (LEFFA; IRALA, 2014, p. 35).

In der deutschsprachigen Fachliteratur spielt jedoch der Begriff der „língua adicional“ bzw. ,,additional language“ als konzeptuelle Kategorie bisher kaum eine Rolle. Was insbesondere die Aneignungskontexte der deutschen Sprache betrifft, so dominieren weiterhin die Bezeichnungen „Deutsch als Fremdsprache“ (DaF) und „Deutsch als Zweitsprache“ (DaZ). Als Erklärung für diese Situation können in erster Linie wissenschaftshistorische, aber auch bildungspolitische 
Gründe angeführt werden, die jedoch ganz anders gelagert sind als in der brasilianischen Diskussion. So hat sich der Fachbereich $\mathrm{DaF}$ in Deutschland ab den 1970er Jahren zu einem grundständigen Studiengang neben der (muttersprachlichen) Germanistik entwickelt und erste Lehrstühle mit der Bezeichnung „Deutsch als Fremdsprache“ wurden eingeführt (vgl. UPHOFF, 2013). Seit den 2000er Jahren ist nun infolge einer immer heterogeneren Zusammensetzung der Gesellschaft ein großer Bedarf an sozialpolitischen Maßnahmen entstanden, der sich spezifisch an diejenigen BürgerInnen richtet, die Deutsch nicht als Erstsprache erworben haben und deshalb auf besondere Hilfestellungen bei Schulbesuchen, Behördengängen und dergleichen angewiesen sind. So kristallisierte sich in relativ kurzer Zeit ein spezifisches Forschungsgebiet für Deutsch als Zweitsprache heraus, das zwar weiterhin viele thematische und konzeptuelle Überschneidungen mit DaF aufweist, jedoch in der scientific community zunehmend als eigenständiger Bereich wahrgenommen wird. In aktuellen Publikationen findet man häufig die Doppelbezeichnung „DaF/DaZ“ bzw. „DaZ/ DaF“, wobei die Reihenfolge der Akronyme meist als Zeichen für die jeweilige Schwerpunktsetzung gewertet werden kann.

Dieser Aspekt war auch bei der Bestimmung des Korpus für die vorliegende Arbeit ausschlaggebend. So wurden Einführungen ausgewählt, die in der letzten Dekade erschienen sind und im Titel die Bezeichnung „Deutsch als Fremdsprache“ führen (RÖSLER, 2012; HUNEKE \& STEINIG, 2013; GEHRING, 2018) bzw. DaF als erste Nennung erwähnen (vgl. den Untertitel „Basiswissen Didaktik. Deutsch als Fremd- und Zweitsprache" von Brinitzer et al., 2016). Werke, die den Bereich DaZ in den Vordergrund stellen (z.B. RÖSCH, 2011; KALKAVAN-AYDIN, 2018) wurden demgegenüber in dieser Arbeit nicht berücksichtigt, sollen aber in einer späteren Studie mit herangezogen werden, um die Existenz unterschiedlicher Wissensordnungen genauer $\mathrm{zu}$ erforschen (cf. UPHOFF, 2019). Eine Sonderstellung nimmt in unserem Korpus das Werk von Roche (2013) ein, dessen Titel nicht spezifisch auf DaF, sondern sprachübergreifend ausgerichtet ist. Der Autor ist jedoch als Professor für Deutsch als Fremdsprache tätig und auch im Buch selbst wird vorwiegend mit deutschsprachigen Beispielen gearbeitet, was die Hinzunahme dieser Einführung rechfertigt.

In der folgenden Sektion möchte ich nun die Wissenssoziologische Diskursanalyse als theoretisch-methodologischen Rahmen für die 
Untersuchung der gewählten Einführungswerke vorstellen.

\section{Wissenssoziologische Diskursanalyse}

Die Wissenssoziologische Diskursanalyse (WDA) ist ein von Reiner Keller begründetes Forschungsprogramm zur Analyse der Entstehung, Legitimierung und Veränderung gesellschaftlicher Wissensverhältnisse und Wissenspolitiken (cf. KELLER, 2011a, 2011b). Es verbindet die Foucaultschen Konzeptionen von Diskurs, Wissen und Macht (KELLER, 2008) mit dem wissenssoziologischen Ansatz von Berger und Luckmann (1966) und betrachtet Wissen als das Ergebnis kollektiver Deutungskämpfe, die von sozialen Gruppen wie z.B. wissenschaftlichen Expertengemeinschaften (scientific communities) über einen bestimmten, in der Gruppe etablierten Kommunikationsmodus ausgetragen werden. Diskurse lassen sich dabei, wie Keller (2011a) formuliert,

als mehr oder weniger erfolgreiche Versuche verstehen, Bedeutungszuschreibungen und Sinn-Ordnungen zumindest auf Zeit zu stabilisieren und dadurch eine kollektiv verbindliche Wissensordnung in einem sozialen Ensemble zu institutionalisieren (KELLER, 2011a, p. 8).

Diskurse stellen ein Konstrukt dar, mit dem postuliert wird, ,dass spezifischen empirischen Daten, die zunächst als singuläre, in Zeit und Raum verstreute Ereignisse (Äußerungen) existieren und dokumentiert sind, ein Zusammenhang, eine Regel oder Struktur unterliegt" (idem, p. 83). Das Ziel der Wissenssoziologischen Diskursanalyse ist es nun, anhand einer bestimmten Fragestellung ein Korpus von Äußerungen zu erstellen und dieses im Hinblick auf die Existenz von regelhaften Sinnzuschreibungen bzw. ihrer Divergenzen zu untersuchen. Dabei steht nicht die Ideologiekritik im Vordergrund, sondern die kritische Untersuchung der Möglichkeitsbedingungen der Diskurse.

Die Regelhaftigkeit von Diskursen kann laut WDA (vgl. KELLER, 2011a, p. 101-112) verschiedene Muster aufweisen, von denen die narrative Struktur oder „storyline“ eine Kategorie darstellt. Diese wurde besonders von Viehöver $(2006,2010,2012)$ untersucht und soll im Folgenden genauer beschrieben werden.

\section{Narrationen als diskursstrukturierende Regelsysteme}

Durch Narrationen werden kleinere Bestandteile einer Aussage zu einer Erzählung oder Geschichte miteinander verbunden. 
Narrative Schemata verleihen den Diskursen dadurch eine spezifische Gestalt und innere Kohärenz, da verstreute Sinnelemente zu einer zusammenhängenden, einheitlichen Geschichte ausgeformt werden (VIEHÖVER, 2012,p. 82). Viehöver(2006,p. 183) sieht in den Narrationen „einen universellen Modus der Kommunikation und der Konstitution von Sinn“. Sie versehen Menschen, wie der Autor (idem, p. 184) weiterhin erläutert, „mit Weltsichten, Motiven, Handlungsorientierungen und kulturellen Werten, indem sie Ereignisse, Objekte, Akteure, Handlungen durch narrative Strukturen konfigurieren oder verknüpfen“.

Für die Analyse von Narrationen differenziert Viehöver (2010, p. 241) insgesamt drei Untersuchungsebenen. Neben der Oberflächenstruktur der sprachlichen Materialität und der Tiefenstruktur der zugrunde liegenden Werte und Weltsichten unterscheidet er eine vermittelnde Ebene, auf der die narrativen Episoden, der Handlungsplot und die Aktantenstruktur der Geschichte angesiedelt sind. Es ist nun besonders diese zwischenliegende Ebene, die in der folgenden Analyse der DaF-Einführungen herausgearbeitet werden soll. Welche Ereignisse, Akteure und Argumentationen werden in den Werken herangezogen, um den Weg vom 20. zum 21. Jahrhundert in der DaF-Didaktik zu beschreiben und plausibel zu machen? Handelt es sich um eher homogene Darstellungen oder fallen verschiedene Erzählweisen ins Auge? Falls letzteres der Fall ist, welche unterschiedlichen Wertestrukturen können in diesen Narrationen vielleicht identifiziert werden?

Wie Viehöver (2010, p. 237) betont, können narrative Schemata „unterschiedliche Funktionen einnehmen, die von der sozialen Integration, der Distinktion sozialer Kollektive, ihrer Mobilisierung bis zur Transformation von Wissensordnungen reichen“. Durch Narrationen versichern sich Diskursgemeinschaften einer gemeinsamen Problemsicht, aus der in der Folge kollektive Lösungswege abgeleitet werden können (VIEHÖVER, 2006, p. 188). Auf der anderen Seite ist aber auch das Potenzial der Veränderung von Wissensregimen gegeben, wenn konkurrierende Narrationen existieren, die auf eine unterschiedliche Problemsicht und eventuell auch divergierende Werte hindeuten. Auch wissenschaftliche Diskurse können, so Viehöver (2010, p. 264), narrative Strukturen nutzen, um ihren Argumentationen Nachdruck zu verleihen 
und bestimmte konzeptuelle Erklärungsansätze kommunikabel und erinnerbar zu machen.

In der nächsten Sektion möchte ich zeigen, dass auch die Methodengeschichte des 20. Jahrhunderts und die didaktischen Entwicklungen zu Beginn des 21. Jahrhunders in den konsultierten DaFEinführungen unterschiedlich erzählt werden, wodurch verschiedene Problemsichten für die Herausforderungen des Deutschunterrichts zum Ausdruck kommen.

\section{DaF im 21. Jahrhundert: Wissensordnungen im Umbruch}

Um die narrativen Strukturen im Korpus zu ermitteln, möchte ich zunächst auflisten, welche „Episoden“, d.h. (globale) Methodenkonzeptionen in der Geschichte des DaF-Unterrichts referiert werden. Es ist interessant zu beobachten, dass sich schon an dieser Stelle unterschiedliche Sichtweisen bzw. Darstellungsformen offenbaren (vgl. Tabelle 1). 
Tabelle 1: beschriebene Methodenkonzeptionen in den Einführungswerken

\begin{tabular}{|c|c|c|c|c|}
\hline Rösler (2012) & $\begin{array}{l}\text { Huneke \& Steinig } \\
\text { (2013) }\end{array}$ & Roche (2013) & $\begin{array}{l}\text { Brinitzer et al. } \\
\text { (2016) }\end{array}$ & Gehring (2018) \\
\hline $\begin{array}{l}\text { Grammatik- } \\
\text { Übersetzungs- } \\
\text { Methode }\end{array}$ & $\begin{array}{l}\text { Grammatik- } \\
\text { Übersetzungs- } \\
\text { Methode }\end{array}$ & $\begin{array}{l}\text { Grammatik- } \\
\text { Übersetzungs- } \\
\text { Methode }\end{array}$ & -- & $\begin{array}{l}\text { Grammatik- } \\
\text { Übersetzungs- } \\
\text { Methode }\end{array}$ \\
\hline-- & -- & - & -- & $\begin{array}{l}\text { Interlinear- } \\
\text { Methode }^{1}\end{array}$ \\
\hline Direkte Methode & -- & -- & -- & -- \\
\hline $\begin{array}{l}\text { Audiolinguale } \\
\text { Methode }\end{array}$ & \multirow{2}{*}{$\begin{array}{l}\text { Audiolinguale/ } \\
\text { Audiovisuelle } \\
\text { Methode }\end{array}$} & $\begin{array}{l}\text { Audiolinguale } \\
\text { Methode }\end{array}$ & -- & $\begin{array}{l}\text { Audiolinguale } \\
\text { Methode }\end{array}$ \\
\hline $\begin{array}{l}\text { Audiovisuelle } \\
\text { Methode }\end{array}$ & & - & -- & -- \\
\hline \multirow{2}{*}{$\begin{array}{l}\text { Kommunikativer } \\
\text { Ansatz }\end{array}$} & \multirow{2}{*}{$\begin{array}{l}\text { Kommunikativ- } \\
\text { pragmatischer } \\
\text { Ansatz }\end{array}$} & \multirow{2}{*}{$\begin{array}{l}\text { Kommunikative } \\
\text { Didaktik und } \\
\text { alternative } \\
\text { Methoden }\end{array}$} & -- & $\begin{array}{l}\text { Total Physical } \\
\text { Response }\end{array}$ \\
\hline & & & -- & $\begin{array}{l}\text { Kommunikativer } \\
\text { Ansatz }\end{array}$ \\
\hline $\begin{array}{l}\text { Interkultureller } \\
\text { Ansatz }\end{array}$ & $\begin{array}{l}\text { Interkultureller } \\
\text { Ansatz }\end{array}$ & $\begin{array}{l}\text { Interkulturelle } \\
\text { Sprachdidaktik }^{2}\end{array}$ & -- & -- \\
\hline $\begin{array}{l}\text { Aufgaben- } \\
\text { orientierung }\end{array}$ & $--^{3}$ & $\begin{array}{l}\text { Handlungs- } \\
\text { und Aufgaben- } \\
\text { orientierung }\end{array}$ & $\begin{array}{l}\text { Handlungs- } \\
\text { und Aufgaben- } \\
\text { orientierung }\end{array}$ & $\begin{array}{l}\text { Aufgabengestützter } \\
\text { Ansatz }\end{array}$ \\
\hline $\begin{array}{l}\text { Alternative } \\
\text { Methoden }\end{array}$ & $--^{4}$ & (siehe oben) & -- & -- \\
\hline $\begin{array}{l}\text { Performatives } \\
\text { Fremdsprachen- } \\
\text { lernen }^{5}\end{array}$ & -- & -- & -- & -- \\
\hline-- & -- & -- & -- & Bilinguale Methode \\
\hline
\end{tabular}

Quelle: eigene Darstellung

1 Laut Gehring (2018, p. 60) ist die Interlinear-Methode „in erster Linie eine Präsentationsmethode, ebenfalls des 19. Jahrhunderts“, die versucht, durch die Spiegelung mutter- und fremdsprachlicher Redemittel Verständnis zu erreichen.

2 Dieser Ansatz wird von Roche (2013) in einem gesonderten Kapitel zu einem späteren Zeitpunkt behandelt.

3 Die Aufgabenorientierung wird im Stichwortverzeichnis erwähnt, es wird ihr in $\mathrm{Hu}-$ neke \& Steinig (2013) jedoch kein gesonderter Abschnitt zugeteilt.

4 Total Physical Resonse wird im Stichwortverzeichnis erwähnt und an anderer Stelle kurz beschrieben.

5 Rösler definiert diese Methodenkonzeption folgendermaßen: „Ein Ansatz, bei dem der ganze Körper konsequent als Teil der Interaktion mit der Umgebung für das Fremdsprachenlernen eingesetzt wird, ist das performative Fremdsprachenlernen, auch bekannt unter den Bezeichnungen ,Fremdsprachenlernen inszenieren“ und, Dramapädagogische Didaktik“" (RÖSLER, 2012, p. 92). 
Tabelle 1 macht deutlich, dass schon bei der Beschreibung der Vergangenheit des DaF-Unterrichts sehr unterschiedliche Erzählstrategien angewendet werden. So beschränken sich Huneke \& Steinig (2013) und Roche (2013) auf eher wenige, zentrale Methodenkonzeptionen, während Rösler (2012) und Gehring (2018) ein differenzierteres Panorama vorstellen. Brinitzer et al. (2016) beleuchten die historische Entwicklung in der Methodendiskussion überhaupt nicht detaillierter und geben sich stattdessen mit einer kurzen und sehr skizzenhaften Bemerkung im einleitenden Teil des Werkes zufrieden:

Wenn Sie an Ihre persönlichen Sprachlernerfahrungen zurückdenken, erinnern Sie sich vielleicht an grammatiklastigen Frontalunterricht, an Vokabeln lernen und Einsetzübungen. Ein solches Verständnis von Unterricht ist jedoch nicht mehr zeitgemäß und entspricht auch nicht den eigentlichen Zielen des Sprachunterrichts (BRINITZER et al., 2016, p. 9).

Diejenigen Autoren, die einen genaueren Blick in die Vergangen heit bieten, halten sich zumeist an eine chronologische Darstellungsweise, die nur bei Rösler (2012) in einem Moment durchbrochen wird, wenn nämlich die alternativen Methoden zusammengefasst in einem eigenen Teilkapitel den globalen Methodenkonzeptionen nachgestellt werden.

Aufschlussreich ist auch die Bezeichnung der Kapitel, in denen die Entwicklung der Methodendiskussion in den einzelnen Werken erzählt wird. Während Rösler(2012) und Huneke \& Steinig (2013) dieseAbschnitte mit „Umfassende Konzepte der Fremdsprachenvermittlung“ bzw. „Globale Methodenkonzeptionen im DaF-Unterricht" betiteln und somit den universalen Charakter der besprochenen Methoden herausstellen, nennt Gehring (2018) sein Kapitel lapidar „Sprachlehrmethoden“, ohne weitere Spezifizierung. Roche (2013) schägt einen ganz anderen Weg ein, indem er sein Kapitel mit „Von der Inputsteuerung zur Kompetenzorientierung“ überschreibt und im geschichtlichen Überblick nicht die methodischen Verfahrensweisen, sondern die ihnen zugrunde liegenden Sprach- und Lernkonzeptionen ins Zentrum stellt.

Einen klaren Hinweis auf die derzeitige Umbruchsituation in der Wissensordnung im DaF-Bereich finden wir nun, wenn wir betrachten, wie die aktuelle didaktisch-methodische Diskussion in den Einführungswerken jeweils beschrieben wird. Hier gibt es erstaunliche Unterschiede, nicht nur in der Bewertung der aktuellen Phase, sondern auch in dem „Plot“ der Erzählung, d.h., nach Viehöver (2012, p. 68), 
in der „konfigurierten Synthese von Charakteren, Handlungen und Ereignissen“, die zu der gegenwärtigen Situation geführt hat.

In zwei der fünf Einführungen wird auf eine schon eingetretene Zäsur zu vergangenen Vermittlungsformen aufmerksam gemacht. ${ }^{1}$ Brinitzer et al. (2016, p. 9) belassen es bei einem kurzen Verweis auf den Gemeinsamen Europäischen Referenzrahmen für Sprachen (EUROPARAT, 2001), der die aktuelle Handlungs- und Aufgabenorientierung im Fremdsprachenunterricht untermauert und markiert. Rösler (2012, p. 65-67) hingegen leitet sein Methodenkapitel recht ausführlich mit einem forschungsgeschichtlichen Kommentar ein, um die seinen Worten nach ,paradoxe Situation“ (idem, p. 76) in der Methodendiskussion des ausgehenden 20. Jahrhunderts zu erklären:

Spätestens ab den 1970er Jahren war klar, dass sich die Fremdsprachendidaktik mit der Suche nach der ,besten` Methode in eine Sackgasse manövriert hatte. [...] Man hätte deshalb erwarten können, dass seit den 1970er Jahren gar keine globale Methodendiskussion mehr stattfindet. Das ist nicht so: Vor allem mit dem kommunikativen Ansatz und zu einem geringeren Maße auch mit dem interkulturellen Ansatz ist die globale Diskussion fortgeführt worden (RÖSLER, 2012, p. 67).

Der Auslöser für diese „Sackgasse“ wird in einem in den historischen Abriss der Methodengeschichte eingeschobenen Teilkapitel („Unabhängigkeit von der Entwicklung der Bezugswissenschaften“; RÖSLER, 2012, p. 75-76) genauer erläutert. Hier erwähnt der Autor zwei große Studien - Smith (1970) und Elek \& Oskarsson (1975) -, die versucht haben, den Beweis der Überlegenheit einer Methode zu erbringen, damit jedoch scheiterten. Trotz dieser empirischen Gegenevidenz, so Rösler, blieb es noch weitere Jahrzehnte bei der „Methodenfixierung“ (RÖSLER, p. 67), wenn auch zunehmend mit vorsichtigeren Begrifflichkeiten wie „Ansatz“ und „Orientierung“" anstatt von „Methode“ (idem, p. 76) operiert wurde. Der von Kumaravadivelu $(2003,2006)$ um die Jahrtausendwende geprägte Begriff der „Post-Methode“ ist nach Rösler (2012, p. 67) lediglich als ein Appell zu werten, diese obsolete Fokussierung auf das Konzept

1 Auch die narrative Struktur von Roche (2013) beinhaltet eine Zäsur, die sich jedoch im Gegensatz zu Rösler (2012) und Brinitzer et al. (2016) noch nicht durchgehend vollzogen hat und deshalb erst im weiteren Verlauf dieser Arbeit besprochen werden soll. 
der globalen Methode nun endlich zu überwinden. An dieser Stelle muss auch hervorgehoben werden, dass Rösler der einzige Autor im Korpus ist, der das Werk von Kumaravadivelu überhaupt erwähnt, was auf eine (immer noch) sehr begrenzte Rezeption der Schriften Kumaravadivelus im Bereich Deutsch als Fremdsprache schließen lässt.

Was nun die Diagnostizierung der aktuellen didaktischmethodischen Praxis im Bereich DaF anbelangt, gibt es Überschneidungen in drei der fünf konsultierten Einführungen, die sich jedoch zum Teil wieder sehr in der Bewertung der derzeitigen Lage voneinander unterscheiden. Rösler (2012), Roche (2013) und auch Gehring (2018) gehen davon aus, dass Versatzstücke verschiedener (globaler) Methoden noch heute von vielen Lehrpersonen verwendet werden. Rösler (2012, p. 66) betont, dass es unangemessen ist, von „veralteten“"vs. „modernen“ Methoden zu sprechen: „Wenn man bestimmte Vorgehensweisen einschätzen möchte, muss man diese in Beziehung zu handelnden Personen, den Lernzielen und den institutionellen Bedingungen setzen“ (idem). Hier manifestiert sich nach Rösler die wichtigste Erkenntnis im Prozess der Überwindung des Methodenparadigmas:

Das aus heutiger Sicht wohl interessanteste Ergebnis dieser beiden Studien [Smith (1970) und Elek \& Oskarsson (1975); siehe oben] war, dass die Frage nach der Überlegenheit einer Methode über andere eigentlich die falsche Frage ist. Fragen nach der Überlegenheit von Vorgehensweisen können nicht auf der Ebene allgemeiner Methoden gestellt werden, sondern müssen sich auf die Vielfalt des Lernens beziehen, auf individuenbezogene Faktoren wie z. B. Alter, Motivation usw. ebenso wie auf Lernziele oder institutionelle Begebenheiten (RÖSLER, 2012, p. 75, Hervorhebung hinzugefügt).

Sprachlernprozesse sind Rösler zufolge also je nach Lerner und Kontextbedingungen unterschiedlich und vielgestaltig, weshalb methodische Verfahren lokal definiert und ausgehandelt werden müssen. Es zeigt sich bei diesem Standpunkt eine große Übereinstimmung mit dem Parameter der particularity von Kumaravadivelu (2003, 2006), auf die jedoch vom Autor nicht explizit hingewiesen wird.

Auch Gehring (2018) identifiziert in der derzeitigen DaF-Didaktik eine Vorgehensweise, die Elemente verschiedener Methodenkonzeptionen in sich vereint: „Heute bevorzugt man freiere Konzepte, die stärker auf eine konkrete Aufgabenstellung ausgerichtet ist. Moderne Lehrkräfte kombinieren Elemente klassischer Methoden mit modernen 
Aufgabenformaten“(GEHRING, 2018, p. 59). Bei seinem Überblick über die bisher entwickelten Sprachlehrmethoden beschreibt er jeweils aktuelle Einsatzmöglichkeiten und fast immer auch bestimmte Kritikpunkte. Nur bei der kommunikativen Didaktik wird keine nachteilige Eigenschaft angeführt und der Autor erklärt stattdessen knapp: „Der Kommunikative Ansatz hat Leitliniencharakter im modernen Fremdsprachenunterricht" (GEHRING, 2018, p. 62).

Allein Roche (2013) bewertet die eklektische Anwendung verschiedener methodischer Bestandteile dezidiert negativ. Er beschreibt den aktuellen Zustand in der ,praktischen Welt des Sprachunterrichts“ als einen „Methodenmix, bei dem die verschiedenen Methoden relativ willkürlich kombiniert und ausprobiert werden" (ROCHE, 2013, p. 32). „Diese jeweils unterschiedlich dosierte Mischung von Methoden beschreibt wahrscheinlich am besten den verbreiteten Zustand des Fremdsprachenunterrichts“, so der Autor weiter (idem). Roches Meinung nach müssen nun die spracherwerblichen Grundlagen des Fremdsprachenunterrichts von Lehrkräften besser reflektiert werden, damit es zu einem „Paradigmenwechsel“ (ROCHE, 2013, p. 14), welcher in der Theorie schon stattgefunden habe, auch in der Unterrichtspraxis kommen könne. Deshalb listet er am Ende seines Einführungswerkes insgesamt 15 Parameter einer ,zukünftigen Fremdsprachendidaktik“ (ROCHE, 2013, p. 311) auf, die jedoch erst noch verwirklicht werden müssen. Die Zäsur ist für ihn also in den Bezugswissenschaften des Fremdsprachenunterrichts schon eingetreten, da diese statt einer Inputsteuerung nun für eine Kompetenzorientierung plädieren, während in der Praxis weiterhin ein „Methodenaktionismus“ (idem) vorherrscht. Roche betont allerdings auch, dass der Weg hin zu einer kompetenzorientierten Unterrichtspraxis kein leichter ist und weitere Qualifizierungsmaßnahmen für Lehrpersonen notwendig sind:

Die Sprachdidaktik ist mit einer Reihe von Aufgaben konfrontiert, bei deren Lösung sie oft einen Balanceakt vollbringen muss. Diese Aufgaben können nicht allein durch methodische Glaubensbekenntnisse oder durch Methodenaktionismus gelöst werden. Vor allem bedarf es einer systematischen Nutzung von Forschungsergebnissen aus der Linguistik, der Lernpsychologie und der Spracherwerbsforschung [...]. Zudem sollten Lehrkräfte etwas mutiger das von ihnen erworbene Expertenwissen nutzen, systematisieren und in die Fachdiskussion einbringen. Dazu bedarfes jedoch einer entsprechenden Sensibilisierung, Ausbildung, Einbindung und Betreuung (ROCHE, 2013, p. 311). 
Im Gegensatz zu Rösler (2012), Roche (2013) und Gehring (2018) wird der historische Überblick über die Methodendiskussion bei Huneke \& Steinig (2013) relativ isoliert und ohne Bezugnahme auf eine aktuelle Situationsbeschreibung dargestellt. So wird lediglich erwähnt, dass die vier besprochenen Methodenkonzeptionen (GrammatikÜbersetzungs-Methode, audiolinguale/audiovisuelle Methode, kommunikativ-pragmatischer Ansatz und interkultureller Ansatz; vgl. Tabelle 1) „weithin als ,etabliert““ (HUNEKE \& STEINIG, 2013, p. 200, einfache Anführungszeichen im Original) gelten und dass „die kritische Reflexion und Revision globaler Methodenkonzeptionen [...] den Fremdsprachenunterricht im Grunde schon immer begleitet" hätten (idem, p. 199). In diesem Werk wird die Möglichkeit einer Mischung verschiedener Methoden also weder explizit angesprochen noch positiv oder negativ konnotiert. Sie verliert damit ihre argumentative Kraft, die besonders in den Narrationen von Rösler (2012) und Roche (2013) eine große Rolle spielt.

Zum Abschluss der Analyse möchte ich mich kurz der Aktantenstruktur innerhalb der methodengeschichtlichen Darstellungen zuwenden und mich dabei besonders auf die Adressierung der Lehrkräfte in den Erzählungen konzentrieren. Die narrativen Strategien sind hier wiederum recht breit gestreut. Während Huneke \& Steinig (2013) und Gehring (2018) eine eher unpersönliche und neutrale Darstellungsform wählen, bei der das didaktisch-methodische Handeln der Lehrpersonen nur beschrieben, aber nicht bewertet wird, zeichnen Brinitzer et al. (2016) schon im einleitenden Teil ihres Werkes ein Bild von einem, guten Lehrer bzw. einer gute[n] Lehrerin“ (idem, p. 10), dem/der sie eine klare methodische Anweisung geben: ,Auch Ihre Lehrtätigkeit sollte von der Handlungs- und Aufgabenorientierung geprägt sein. Bei der Unterrichtsplanung ist somit eine Orientierung an den Zielen des Sprachhandelns ausschlaggebend und nicht die Ausrichtung an grammatischen Phänomenen“ (idem). Hier wird schon zu Beginn der Einführung ein positives Modell einer Lehrkraft skizziert, dem die Leserinnen und Leser nachstreben sollen. Wie wir in unseren bisherigen Ausführungen schon gesehen haben, ist es bei Roche (2013) genau andersherum. Er nämlich konstruiert ein eher negatives Bild der Lehrperson, die unterschiedliche Methoden willkürlich und wenig reflektiert einsetzt und dadurch einen fragwürdigen „Methodenaktionismus“ (idem, p. 311) praktiziert. Im Unterschied dazu zeigt sich Rösler (2012) bemüht, mögliche Vorurteile gegenüber 
einem scheinbar mit veralteten Mitteln gestalteten Deutschunterricht auszuräumen, indem er die Kontextbedingtheit der adäquaten Methodenwahl unterstreicht. Er verweist aber auch darauf, dass „im Alltag der Lehre [...] eine kritische Auseinandersetzung " mit den globalen Methodenkonzeptionen „unerlässlich ist“ (RÖSLER, 2012, p. 2) und damit Elemente verschiedener Methoden nicht unreflektiert eingesetzt werden sollten.

Zusammenfassend lässt sich also sagen, dass die fünf untersuchten Einführungwerke den aktuellen Moment in der DaF-didaktischen Diskussion und die Entwicklungen vom 20. ins 21. Jahrhundert sehr unterschiedlich beschreiben und bewerten. Die Methodengeschichte wird mal sehr ausführlich (RÖSLER, 2012), mal eher kompakt (HUNEKE \& STEINIG, 2013; GEHRING, 2018) und in einem Fall (BRINITZER et al., 2016) sogar überhaupt nicht mehr erzählt, sondern auf eine kurze und sehr schematische Gegenüberstellung von zwei Episoden (früher und heute) heruntergebrochen. Auch die Ereignisse, die einen möglichen Wendepunkt zu Beginn des 21. Jahrhundert auslösen, werden, wie wir gesehen haben, unterschiedlich markiert bzw. in den Werken von Huneke \& Steinig (2013) und Gehring (2018) gar nicht identifiziert. Das Konzept der Post-Methoden-Ära spielt in keiner der konsultierten Einführungen eine Rolle und nur Rösler (2012) erwähnt die Schriften von Kumaravadivelu $(2003,2006)$ überhaupt. Auch was die Beurteilung der Kombination verschiedener Methodenelemente im Unterricht betrifft, gibt es unterschiedliche Ansichten. Roche (2013) unterstellt den Lehrkräften dabei mangelnde theoretische Reflexion, während Gehring (2018) diese Handlungsweise schlichtweg als übliche Praxis darstellt und Rösler (2012) die Lehrenden sogar indirekt in Schutz nimmt, wenn er von der Notwendigkeit spricht, den Fremdsprachenunterricht an den jeweils geltenden kontextuellen Bedingungen auszurichten. Ein relativer Konsens besteht in der Benennung der Aufgabenorientierung - zum Teil gepaart mit der Handlungsorientierung - als eine der neuesten Entwicklungen in der DaF-Didaktik, wie Tabelle 1 zeigt. Doch nur bei Brinitzer et al. (2016) wird dieser Ansatz als alleiniges Kernkonzept des modernen Fremdsprachenunterrichts behandelt. Es zeigt sich also ein sehr heterogenes Bild der aktuellen Wissensordnung(en) im DaF-Bereich, das auf eine Umbruchssituation in der methodisch-didaktischen Diskussion schließen lässt. 


\section{Ausblick}

Wie lassen sich nun diese Ergebnisse für die DaF-Lehrerbildung in Brasilien verwerten? Welche Geschichte der Unterrichtspraktiken sollten wir als DozentInnen z.B. unseren Studierenden in der licenciatura erzählen? Müssen wir überhaupt noch genauer in die Vergangenheit schauen oder reichen kurze und unspezifische Angaben wie bei Brinitzer et al. (2016)? Und wie ist es mit dem Konzept der Post-Methode, welches in hiesigen Narrationen zur Geschichte des Fremdsprachenunterrichts eine wichtige Rolle spielt (vgl. LEFFA, 2012; LEFFA \& IRALA, 2014), in der deutschsprachigen DaF-Literatur jedoch kaum rezipiert wird? Um auf diese Fragen Antwort zu geben, halte ich eine grundsätzliche Positionierung zur Bedeutung der Kontextfaktoren (particularity) wie auch des Entscheidungsfreiraumes (practicality), der Fremdsprachenlehrkräften zur Bestimmung der Unterrichtsverfahren zugestanden werden sollte, für notwendig. In Uphoff (2018) habe ich für deshalb für eine kritische und breit angelegte methodisch-didaktische Grundausbildung in der licenciatura plädiert, in der auch der Blick in die Vergangenheit einen wichtigen Beitrag dazu leistet, die Bedeutung eines kontextsensiblen Fremdsprachenunterrichts aufzuzeigen und ein kritisches pädagogisches Urteilsvermögen bei den neuen Lehrergenerationen zu fördern.

\section{LITERATURVERZEICHNIS}

BERGER, Peter L.; LUCKMANN, Thomas. [1966]. A construção social da realidade. $19^{\mathrm{a}}$ ed. Petrópolis: Vozes, 2000.

BRINITZER, Michaela; HANTSCHEL, Hans-Jürgen; KROEMER, Sandra; MÖLLER-FRORATH, Monika; ROS, Lourdes. DaF unterrichten. Basiswissen Didaktik Deutsch als Fremd- und Zweitsprache. Stuttgart: Klett, 2016.

ELEK, Tibor von; OSKARSSON, Mats (orgs.). Comparative method experiments in foreign language teaching. Göteburg: Department of Educational Research, School of Education, 1975.

EUROPARAT. Gemeinsamer europäischer Referenzrahmen für Sprachen: Lernen, lehren, beurteilen. München: Langenscheidt, 2001.

GEHRING, Wolfgang. Fremdsprache Deutsch unterrichten. Bad Heilbronn: Julius Klinkhardt, 2018. 
GERLACH, David; LEUPOLD, Eynar. Kontextsensibler Fremdsprachenunterricht. Tübingen: Narr Francke Attempto, 2019.

HUNEKE, Hans-Werner; STEINIG, Wolfgang. Deutsch als Fremdsprache. Eine Einführung. 6., neu bearbeitete und erweiterte Auflage. Berlin: Erich Schmidt Verlag, 2013.

KALKAVAN-AYDIN, Zeynep (org.). DaZ/DaF Didaktik. Praxishandbuch für die Sekundarstufe I und II. Berlin: Cornelsen, 2018.

KELLER, Reiner. Michel Foucault. Konstanz: UVK, 2008.

KELLER, Reiner. Diskursforschung. Eine Einführung für SozialwissenschaftlerInnen. 4. Aufl. Wiesbaden: VS Verlag, 2011a.

KELLER, Reiner. Wissenssoziologische Diskursanalyse. Grundlegung eines Forschungsprogramms. 3. Aufl. Wiesbaden: Springer VS, $2011 \mathrm{~b}$.

KUMARAVADIVELU, B. Beyond Methods: Macrostrategies for Language Teaching. New Have: Yale University, 2003.

KUMARAVADIVELU, B. Understanding Language Teaching. From Method to Postmethod. New York: Routledge, 2006.

LEFFA, Vilson. Ensino de línguas: passado, presente e futuro. Revista Estudos da Linguagem, v. 20, n. 2, p. 389-411, 2012.

LEFFA, Vilson; IRALA, Valesca Brasil. O ensino e outra(s) língua(s) na contemporaneidade: questões conceituais e metodológicas. In: LEFFA, Vilson; IRALA, Valesca Brasil (orgs.). Uma espiadinha na sala de aula: ensinando línguas adicionais no Brasil. Pelotas: Educat, 2014, p. 21-48.

ROCHE, Jörg. Fremdsprachenerwerb, Fremdsprachendidaktik. 3., vollständig überarbeitete Auflage. Tübingen: Narr Francke Attempto, 2013.

RÖSCH, Heidi. Deutsch als Zweit- und Fremdsprache. Berlin: Akademie Verlag, 2011.

RÖSLER, Dietmar. Deutsch als Fremdsprache. Eine Einführung. Stuttgart: Metzler, 2012.

SCHLATTER, Margarete; GARCEZ, Pedro. Línguas Adicionais (Espanhol e Inglês). In: SECRETARIA DA EDUCAÇÃO DO ESTADO DO RIO GRANDE DO SUL. Referencial Curricular. Lições do Rio Grande, v. 1. Porto Alegre: SEDUC, 2009, p. 127;172. Disponível em: <https://servicos.educacao.rs.gov.br/ dados/refer curric voll.pdf $>$. Acesso em: 27 jan. 2020.

SMITH, Philip (org.). A comparison of the cognitive and audiolingual approaches to foreign language instruction. The Pennsylvania Foreign Language Project. Philadelphia: Center for Curriculum Development, 1970. 
UPHOFF, Dörthe. A área de Alemão como Língua Estrangeira: desenvolvimento histórico e perspectivas atuais. Pandaemonium Germanicum, v. 16, n. 22, p. 219-241, 2013. Disponível em: <http://www.revistas.usp.br/pg/article/ view/80112/83994>. Acesso: 14 jan.2020.

UPHOFF, Dörthe. O lugar da criticidade na formação inicial de professores de alemão. In: FERRAZ, Daniel de Mello; KAWACHI-FERRAZ, Claudia Jotto (orgs.). Educação linguística em línguas estrangeiras. Campinas: Pontes Editores, 2018, p. 231-247.

UPHOFF, Dörthe. Projeto de pesquisa Print-CAPES. Metodologia de ensino de alemão: regimes de saber e narrativas históricas. São Paulo: DLM/FFLCH/ USP, 2019.

VIEHÖVER, Willy. Diskurse als Narrationen. In: KELLER, Reiner et al. (orgs.). Handbuch Sozialwissenschaftliche Diskursanalyse. Bd. 1: Theorien und Methoden. 2. Aufl. Opladen: Leske + Budrich, 2006, p. 179-208.

VIEHÖVER, Willy. Die Wissenschaft und die Wiederverzauberung des sublunaren Raumes. Der Klimadiskurs im Licht der narrativen Diskursanalyse. In: KELLER, Reiner et al. (orgs.). Handbuch sozialwissenschaftliche Diskursanalyse. Band 2: Forschungspraxis. 4. Aufl. Wiesbaden: VS Verlag für Sozialwissenschaften, 2010, p. 233-269.

VIEHÖVER, Willy. "Menschen lesbarer machen": Narration, Diskurs, Referenz. In: ARNOLD, Markus; DRESSEL, Gert; VIEHÖVER, Willy (orgs.). Erzählungen im Öffentlichen. Über die Wirkung narrativer Diskurse. Wiesbaden: Springer VS, 2012, p. 65-132.

WEINGART, Peter. Wissenschaftssoziologie. Bielefeld: transcript Verlag, 2003.

Recebido em: 31/07/2019

Aceito em: 30/11/2019 